\title{
Peakon, Cuspon, Compacton, and Loop Solutions of a Three-Dimensional 3DKP $(3,2)$ Equation with Nonlinear Dispersion
}

\author{
Haixia Zhao, Lijing Qiao, and Shengqiang Tang \\ School of Mathematics and Computing Science, Guilin University of Electronic Technology, Guilin, Guangxi 541004, China \\ Correspondence should be addressed to Shengqiang Tang; tangsq@guet.edu.cn
}

Received 15 April 2014; Accepted 13 July 2014; Published 13 August 2014

Academic Editor: Mohammad T. Darvishi

Copyright (C) 2014 Haixia Zhao et al. This is an open access article distributed under the Creative Commons Attribution License, which permits unrestricted use, distribution, and reproduction in any medium, provided the original work is properly cited.

We study peakon, cuspon, compacton, and loop solutions for the three-dimensional Kadomtsev-Petviashvili equation (3DKP(3, 2 ) equation) with nonlinear dispersion. Based on the method of dynamical systems, the $3 \mathrm{DKP}(3,2)$ equation is shown to have the parametric representations of the solitary wave solutions such as peakon, cuspon, compacton, and loop solutions. As a result, the conditions under which peakon, cuspon, compacton, and loop solutions appear are also given.

\section{Introduction}

Nonlinear phenomena play a crucial role in applied mathematics and physics. Studies of various physical structures of nonlinear dispersive equations had attracted much attention in connection with the important problems that arise in scientific applications. Mathematically, these physical structures have been studied by using various powerful and efficient methods, such as inverse scattering method [1], Darboux transformation method [2,3], Hirota bilinear method [4], Lie group method $[5,6]$, bifurcation method of dynamic systems $[7,8]$, tanh function method [9-12], Fan-expansion method $[13,14]$, and homogenous balance method [15]. Practically, there is no unified technique that can be employed to handle all types of nonlinear dispersive equations.

Recently, Xie and Yan [16] considered the following threedimensional Kadomtsev-Petviashvili equation with nonlinear dispersion (the $3 \mathrm{DKP}(m, n)$ equations in short):

$$
\left[u_{t}+a\left(u^{m}\right)_{x}+\left(u^{n}\right)_{x x x}\right]_{x}+(u)_{y y}+(u)_{z z}=0,
$$

where $a$ is a nonzero real number.

When $n=1, m=2,3 \operatorname{DKP}(2,1)$ equation becomes the 3DKP equation $[17,18]$

$$
\left[u_{t}+2 a u u_{x}+u_{x x x}\right]_{x}+(u)_{y y}+(u)_{z z}=0 .
$$

When $n=1, m=3,3 \operatorname{DKP}(3,1)$ equation becomes the 3DmKP equation

$$
\left[u_{t}+3 a u u_{x}^{2}+u_{x x x}\right]_{x}+(u)_{y y}+(u)_{z z}=0 .
$$

When $a=1 / 2, m=n=2$, Rosenau and Hyman [19] have given a compacton solution to $3 \mathrm{DKP}(2,2)$ equation

$$
\left[u_{t}+u u_{x}+\left(u^{2}\right)_{x x x}\right]_{x}+(u)_{y y}+(u)_{z z}=0 .
$$

By using some transformations, some compactons and solitary patterns are obtained [16]. Recently, by using the ansatz method and the Exp-function method, Inc [20] considered some compact and noncompact solutions for (1) and obtained a new traveling wave solution for the $3 \mathrm{DKP}(2,1)$ equation. The authors did not study the bifurcation behavior of the traveling wave solutions of the corresponding traveling wave equations in its parameter space. It is important to understand the dynamical behavior for the traveling wave solutions governed by a singular traveling wave equation. We emphasize that peakon, cuspon, and loop solutions have not been available as yet. To answer this question, we will consider the bifurcations of traveling wave solutions of $3 \mathrm{DKP}(3,2)$ equation in the three-parameter space $(\alpha, r, \beta)$. 
We now assume that $m=3, n=2$ and make the transformations $u(x, y, z, t)=\phi(k(x+l y+s z-\lambda t))=\phi(\xi)$, where $k, l, s, \lambda \neq 0$. Thus, (1) becomes

$$
\left[-\lambda \phi^{\prime}+a\left(\phi^{3}\right)^{\prime}+k^{2}\left(\phi^{2}\right)^{\prime \prime \prime}\right]^{\prime}+\left(l^{2}+s^{2}\right) \phi^{\prime \prime}=0,
$$

where " $"$ " is the derivative with respect to $\xi$. Integrating (5) twice, we have

$$
\alpha\left(g+\beta \phi+\phi^{3}\right)+2\left(\phi^{\prime}\right)^{2}+2 \phi \phi^{\prime \prime}=0
$$

where $g$ is an integration constant, $\alpha=a / k^{2}$, and $\beta=$ $(1 / a)\left(l^{2}+s^{2}-\lambda\right)$. We further make the transformation

$$
\psi=\phi(\xi)-r, \quad \xi=\xi, \quad y=y,
$$

where $r$ satisfies the equation $\phi^{3}+\beta \phi+g=0$. Then (6) is equivalent to the following two-dimensional systems:

$$
\begin{gathered}
\frac{d \psi}{d \xi}=y \\
\frac{d y}{d \xi}=-\frac{1}{2(\psi-r)}\left[2 y^{2}+\alpha \psi\left(\psi^{2}+3 r \psi+3 r^{2}+\beta\right)\right],
\end{gathered}
$$

which admits the following first integral:

$$
\begin{aligned}
y^{2}= & -\frac{\alpha}{(\psi-r)^{2}} \\
& \times\left(\frac{1}{5} \psi^{5}+\frac{1}{2} r \psi^{4}+\frac{1}{3} \beta \psi^{3}-\frac{r}{2}\left(3 r^{2}+\beta\right) \psi^{2}+h\right), \\
H(\psi, y)= & -\frac{1}{\alpha}(\psi-r)^{2} y^{2}-\psi^{2} \\
& \times\left(\frac{1}{5} \psi^{3}+\frac{1}{2} r \psi^{2}+\frac{1}{3} \beta \psi-\frac{r}{2}\left(3 r^{2}+\beta\right)\right) \\
= & h .
\end{aligned}
$$

Systems (8) are planar dynamical systems defined in the 3 -parameter space $(\alpha, r, \beta)$. For a fixed $\alpha$, we will investigate the bifurcations of phase portraits of (8) in the phase plane $(\psi, y)$ as the parameters $r, \beta$ are changed.

We emphasize that when $\psi=r$, the right-hand side of the second equation of systems (8) is discontinuous. We call such systems the singular traveling wave systems. The straight line $\psi=r$ in the $\psi$ - $y$-phase plane is called a singular straight line. There are some general theories and methods for investigating this type of singular traveling wave systems [2125]. It is now well known that the existence of the singular straight lines implies the occurrence of some nonsmooth dynamical behaviors and curve breaking phenomena of the traveling wave solutions of such system, more precisely the so-called peakon and cuspon, and so forth.

It is important to understand the dynamical behavior for the traveling wave solutions governed by a singular traveling wave equation.
The rest of this paper is organized as follows. In Section 2, we discuss the bifurcations of phase portraits of (8), where explicit parametric conditions will be derived. In Section 3, we give explicit parametric representations for peakon, cuspon, compacton, and loop solutions of (1). Section 4 contains the concluding remarks.

\section{Bifurcation Set and All Phase Portraits of System (8)}

Throughout we assume that $\alpha<0$. Otherwise, we can make a transformation

$$
\begin{array}{ccc}
\alpha \longrightarrow-\alpha, & y \longrightarrow-y, & \psi \longrightarrow-\psi \\
\zeta \longrightarrow-\zeta, & r \longrightarrow-r, \quad \beta \longrightarrow \beta
\end{array}
$$

to reduce (8) to this case. Based on $\psi=r$ being a straight line solution to the system

$$
\frac{d \psi}{d \zeta}=(\psi-r) y
$$

$$
\frac{d y}{d \zeta}=-2 y^{2}-\alpha \psi\left(\psi^{2}+3 r \psi+3 r^{2}+\beta\right),
$$

where $d \xi=(\psi-r) d \zeta$, for $\psi \neq r$, we say that the system (12) is the associated regular system of (8) [25].

Thus, system (12) has five equilibrium points $O(0,0)$, $A_{ \pm}\left(\psi_{ \pm}, 0\right)$, and $S_{ \pm}(r, \pm \sqrt{Y})$, where

$$
\begin{gathered}
\psi_{ \pm}=\frac{1}{2}(-3 r \pm \sqrt{\Delta}), \quad \Delta=-3 r^{2}-4 \beta>0, \\
Y=-\frac{1}{2} r \alpha\left(7 r^{2}+\beta\right)>0 .
\end{gathered}
$$

Let $M\left(\psi_{e}, y_{e}\right)$ be the coefficient matrix of the linearized system of (12) at an equilibrium point $\left(\psi_{e}, y_{e}\right)$ and let $J\left(\psi_{e}, y_{e}\right)$ be its Jacobin determinant. Then, we have

$$
J\left(\psi_{e}, y_{e}\right)=-8 y_{e}^{2}+2 \alpha\left(\psi_{e}-r\right)\left(3 \psi_{e}^{2}+6 r \psi_{e}+3 r^{2}+\beta\right) .
$$

By the theory of planar dynamical systems, we know that for an equilibrium point $\left(\psi_{e}, y_{e}\right)$ of a planar integrable system, if $J<0$, then the equilibrium point is a saddle point; if $J>0$ and Trace $\left(M\left(\psi_{e}, y_{e}\right)\right)=0$, then it is a center point; if $J>0$ and $\left(\operatorname{Trace}\left(M\left(\psi_{e}, y_{e}\right)\right)\right)^{2}-4 J\left(\psi_{e}, y_{e}\right)>0$, then it is a node; and if $J=0$ and the Poincare index of the equilibrium point is zero, then it is a cusp.

For the Hamiltonian defined by (10), we write that

$$
\begin{aligned}
h_{ \pm} & =H\left(\psi_{ \pm}, 0\right) \\
& =-\left(\psi_{ \pm}\right)^{2}\left[\left(-\frac{3}{10} r^{2}+\frac{2}{15} \beta\right) \psi_{ \pm}-\frac{2}{5} r\left(3 r^{2}+\beta\right)\right], \\
h_{s} & =H(r, \pm \sqrt{Y})=r^{3}\left(\frac{4}{5} r^{2}+\frac{1}{6} \beta\right), \quad h_{0}=H(0,0)=0 .
\end{aligned}
$$




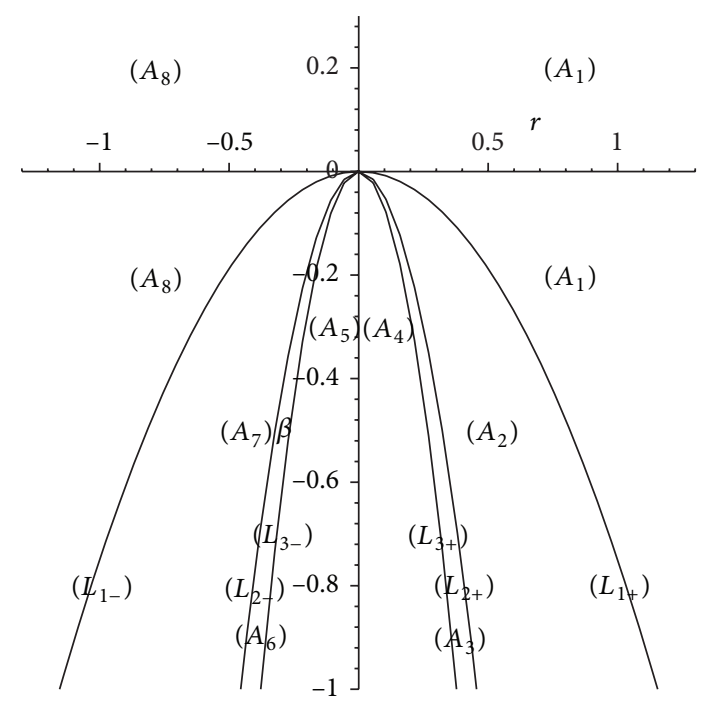

FIGURE 1: The bifurcation set of system $(8)$ in $(r, \beta)$-parameter plane for $\alpha<0$.

The relations $\Delta=0, H(r, \pm \sqrt{Y})=H(0,0)$, and $Y=0$ give the following three bifurcation curves:

$$
\begin{gathered}
\left(L_{1}\right): \beta=-\frac{3}{4} r^{2} ; \quad\left(L_{2}\right): \beta=-\frac{24}{5} r^{2} ; \\
\left(L_{3}\right): \beta=-7 r^{2} .
\end{gathered}
$$

Thus, in the $(r, \beta)$-plane, we have 8 different parameter regions partitioned by the curves $\left(L_{i}\right), i=1,2,3$, and $r=0$, which are shown in Figure 1.

We use Figure 2 to show the bifurcations of the phase portraits of (12) for $\alpha<0$.

\section{Explicit Parametric Representations of Peakon, Cuspon, Compacton, and Loop Solutions of (8)}

In this section, we give some parametric representations of peakon, cuspon, compacton, and loop solutions. To discuss the existence of peakon and cuspon solutions, we need to use the following two lemmas related to the singular straight line (see [25]).

Lemma 1 (the rapid-jump property of the derivative near the singular straight line). Suppose that in a left (or right) neighborhood of a singular straight line there exists a family of periodic orbits. Then, along a segment of every orbit near the straight line, the derivative of the wave function jumps down rapidly on a very short time interval.

Lemma 2 (existence of finite time interval of solution with respect to wave variable in the positive or negative direction). For a singular nonlinear traveling wave system of the first class with possible change of the wave variable, if an orbit transversely intersects with a singular straight line at a point or it approaches a singular straight line, but the derivative tends to infinity, then it only takes a finite time interval to make moved point of the orbit arrive on the singular straight line.

In the following, we give explicit parametric representations of peakon, cuspon, compacton, and loop solutions.

3.1. Peakon. (1) Suppose that $\alpha<0,(r, \beta) \in L_{1+}$. In this case, we have the phase portrait of (8) shown in Figure 2(i). Notice that $H\left(\psi_{ \pm}, 0\right)=H(-(3 / 2) r, 0)=H(r, \pm \sqrt{Y})=(27 / 40) r^{5}$. We see from (10) that two arch curves connecting $S_{ \pm}$in the left side of the straight line $\psi=r$ have the algebraic equation

$$
y^{2}=-\frac{\alpha}{5}\left(\psi+\frac{3}{2} r\right)^{3} .
$$

Thus, by Lemma 2, we can take initial value as $\psi(0)=0$. Then, we have

$$
\psi(\xi)=-\frac{3}{2} r+\frac{4}{[2 \sqrt{2 / 5 r}+\sqrt{-\alpha / 5}|\xi|]^{2}},
$$

which is a a solitary peaked wave solution to (1) (so called "peakon" [26]). The profile of peakon soliton solution is shown in Figure 3(a).

(2) Suppose that $\alpha<0,(r, \beta) \in L_{2+}$. In this case, we have the phase portrait of $(8)$ shown in Figure $2(\mathrm{k})$. Notice that $H\left(\psi_{ \pm}, 0\right)=H(0,0)=0$. We see from (10) that two arch curves connecting $S_{ \pm}$in the left side of the straight line $\psi=r$ have the algebraic equation

$$
y^{2}=-\frac{\alpha}{5} \psi^{2}\left(\psi+\frac{9}{2} r\right) .
$$

Thus, by Lemma 2 , we can take initial value as $\psi(0)=r$. Then, we have

$$
\psi(\xi)=\frac{9 r}{2}\left[\operatorname{coth}^{2}\left(\operatorname{coth}^{-1} \sqrt{\frac{11}{9}}-\frac{3}{2} \sqrt{-\frac{r \alpha}{10}}|\xi|\right)\right],
$$

which is a peakon soliton solution to (1). The profile of peakon soliton solution is shown in Figure 3(b).

Therefore, we have the following.

Theorem 3. (1) When the parameter groups $\alpha, r, \beta$ of system (8) satisfy the condition $\alpha<0$ with $r>0, \beta=-(3 / 4) r^{2}$, there exists a heteroclinic loop of system (12) given by three branches of the curves $H(\psi, y)=(27 / 40) r^{5}\left(=h_{s}\right)$. As the limit curves of a family of periodic orbits of system (8), the curve triangle (i.e., heteroclinic loop) in Figure 2(i) gives rise to a solitary peaked wave solution (a peakon) of (1), which has the exact parametric representation given by (18).

(2) When the parameter groups $\alpha, r, \beta$ of system (8) satisfy the condition $\alpha<0$ with $r>0, \beta=-(24 / 5) r^{2}$, there exists $a$ heteroclinic loop of system (12) given by three branches of the curves $H(\psi, y)=0$. As the limit curves of a family of periodic orbits of system (8), the curve triangle (i.e., heteroclinic loop) in Figure 2(k) gives rise to a peakon soliton solution to (1), which has the exact parametric representation given by (20).

Remark 4. To the best of our knowledge, solutions (18) and (20) obtained for (1) have not been reported in the literature. 


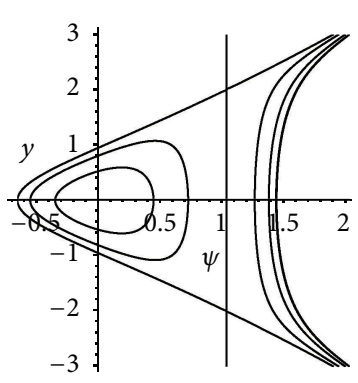

(a) $(r, \beta) \in A_{1}$

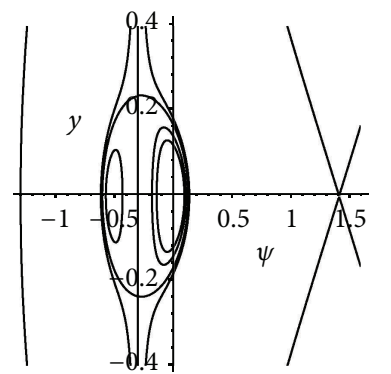

(e) $(r, \beta) \in A_{5}$

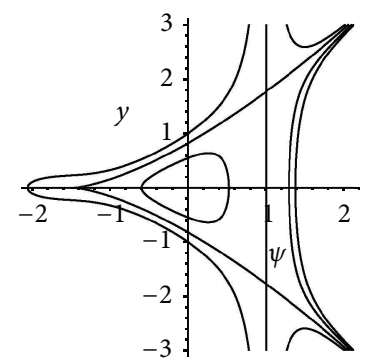

(i) $(r, \beta) \in L_{1+}$

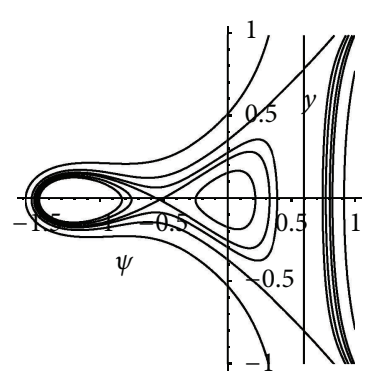

(b) $(r, \beta) \in A_{2}$

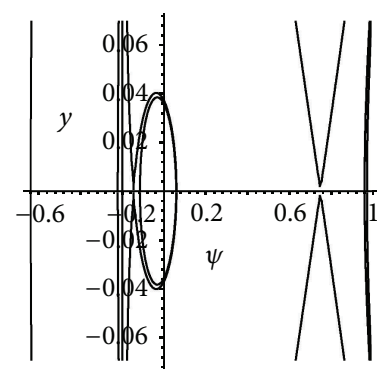

(f) $(r, \beta) \in A_{6}$

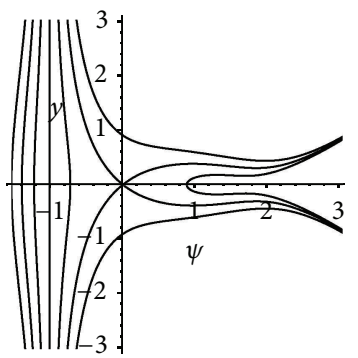

(j) $(r, \beta) \in L_{1-}$

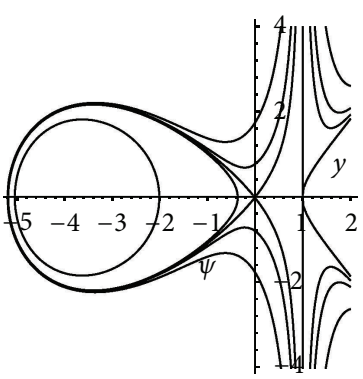

(m) $(r, \beta) \in L_{3+}$

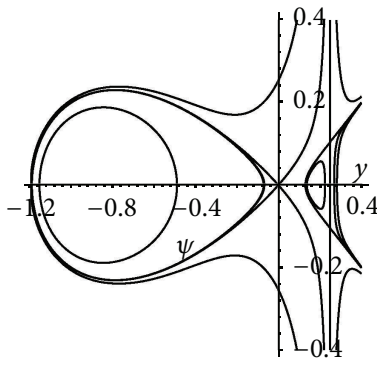

(c) $(r, \beta) \in A_{3}$

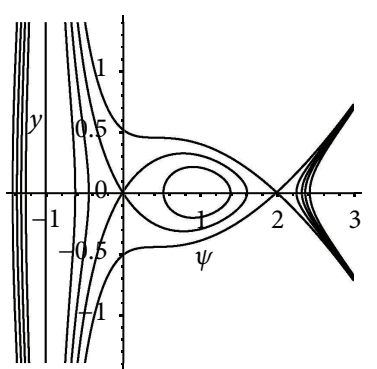

(g) $(r, \beta) \in A_{7}$

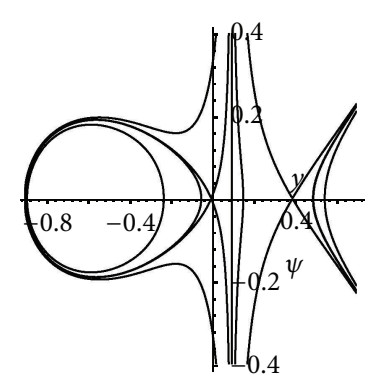

(d) $(r, \beta) \in A_{4}$

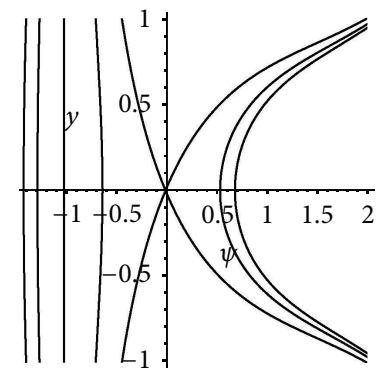

(h) $(r, \beta) \in A_{8}$

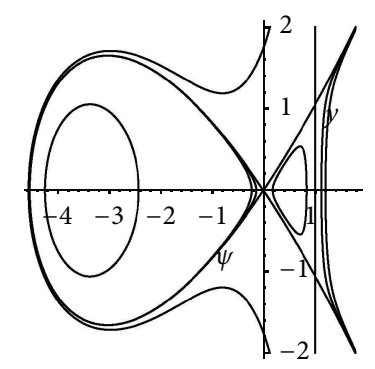

(k) $(r, \beta) \in L_{2+}$

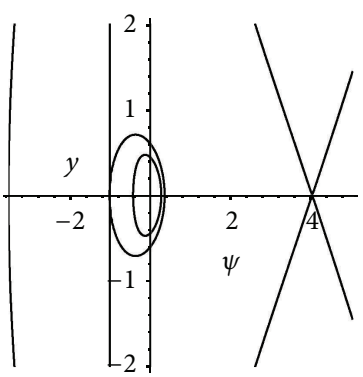

(n) $(r, \beta) \in L_{3-}$

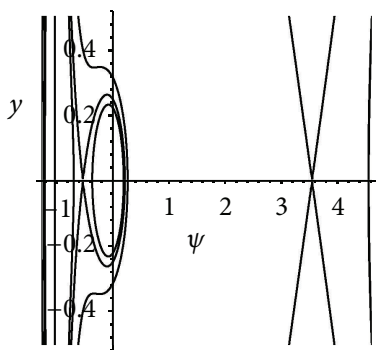

(l) $(r, \beta) \in L_{2-}$

FIgURE 2: The phase portraits of (8) for $\alpha<0$.

3.2. Cuspon. (3) Suppose that $\alpha<0,(r, \beta) \in L_{3+}$. In this case, we have the phase portrait of $(8)$ shown in Figure $2(\mathrm{~m})$. Notice that $H(0,0)=0$. We see from (10) that two arch curves connecting $O(0,0)$ in the right side of the straight line $\psi=r$ have the algebraic equation

$$
y^{2}=-\frac{\alpha \psi^{2}}{5(\psi-r)^{2}}\left(\psi-\psi_{0}\right)\left[\left(\psi+\frac{1}{2}\left(\psi_{0}+\frac{5}{2} r\right)\right)^{2}+\delta^{2}\right],
$$

where $\psi_{0}<\psi_{-}$and $\delta^{2}=\left(-1 / 4 \psi_{0}\right)\left((5 / 2) r \psi_{0}^{2}+(215 / 12) r^{2} \psi_{0}+\right.$ $\left.30 r^{3}\right)$.

On the basis of Lemma 2 , we can take initial value $\psi(0)=$ $r$. Then, we have

$\xi=\sqrt{-\frac{5}{\alpha}} \int_{\psi}^{r} \frac{(\psi-r) d \psi}{\psi \sqrt{\left(\psi-\psi_{0}\right)\left[\left(\psi+(1 / 2)\left(\psi_{0}+(5 / 2) r\right)\right)^{2}+\delta^{2}\right]}}$. 


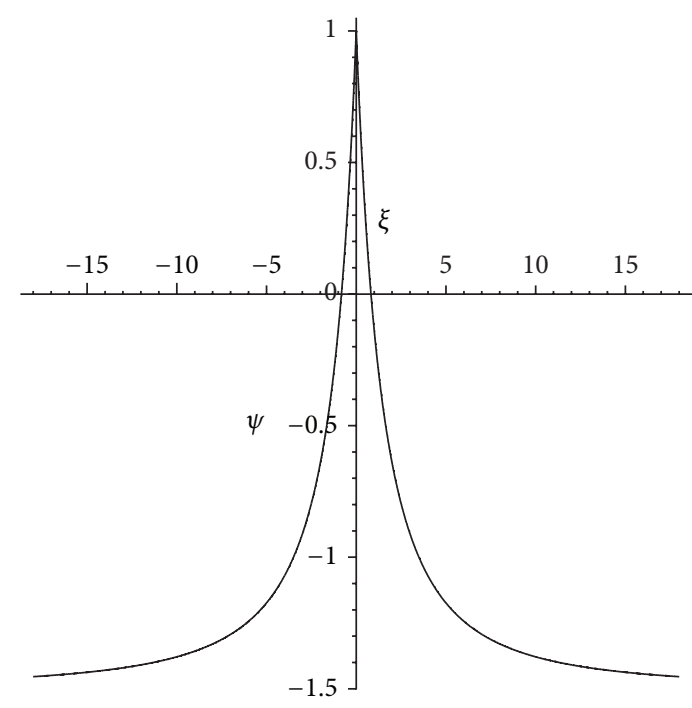

(a) $(r, \beta) \in L_{1+}$

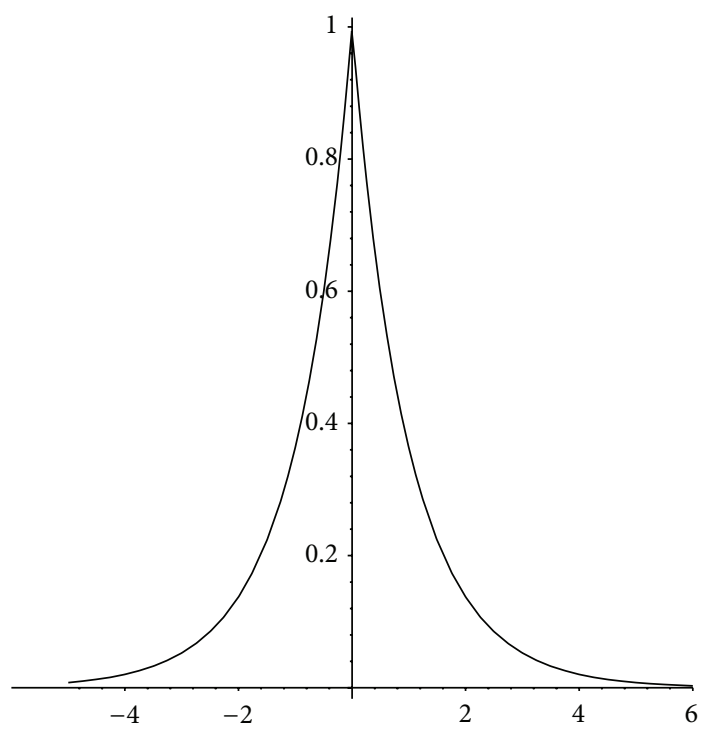

(b) $(r, \beta) \in L_{2+}$

FIgURE 3: The peakon solutions of (8) for $\alpha<0$.

By introducing a new variable $\chi$, from (22), one obtains the parametric representation of the following cuspon solution to (1)

$$
\begin{aligned}
\psi(\chi)=\frac{A+\psi_{0}-\left(A-\psi_{0}\right) \mathrm{cn}(\chi, k)}{1+\operatorname{cn}(\chi, k)}, \\
\chi \in(-\infty, 0], \chi \in[0, \infty), \text { respectively, } \\
\xi(\chi)=\frac{\left(A-\psi_{0}+r\right) g}{A-\psi_{0}} \\
\quad \times \sqrt{-\frac{\alpha}{5}}\left(\chi-\Psi_{1}(\chi)-\chi_{0}+\Psi_{1}\left(\chi_{0}\right)\right),
\end{aligned}
$$

where

$$
\begin{aligned}
\Psi_{1}(\chi)= & \frac{2 r A}{\left(\psi_{0}+A\right)\left(1-\alpha_{1}^{2}\right)} \\
& \times\left(\Pi\left(\arccos (\operatorname{cn}(\chi, k)), \frac{\alpha_{1}^{2}}{\alpha_{1}^{2}-1}, k\right)-\alpha_{1} f_{1}\right), \\
\chi_{0}= & \mathrm{cn}^{-1}\left(\frac{A+\psi_{0}-r}{A-\psi_{0}+r}, k\right), \quad \alpha_{1}=\frac{A-\psi_{0}}{A+\psi_{0}}, \\
& k^{2}=\frac{A+b_{1}-\psi_{0}}{2 A}, \quad g=\frac{1}{\sqrt{A}},
\end{aligned}
$$

$$
\begin{aligned}
f_{1}= & \frac{1}{2} \sqrt{\frac{\alpha_{1}^{2}-1}{k^{2}+k^{\prime 2} \alpha_{1}^{2}}} \\
& \times \ln \left[\frac{\sqrt{k^{2}+k^{\prime 2} \alpha_{1}^{2}} \operatorname{dn}(\chi, k)+\sqrt{\alpha_{1}^{2}-1} \operatorname{sn}(\chi, k)}{\sqrt{k^{2}+k^{\prime 2} \alpha_{1}^{2}} \operatorname{dn}(\chi, k)-\sqrt{\alpha_{1}^{2}-1} \operatorname{sn}(\chi, k)}\right], \\
A & =\sqrt{\left(-\frac{1}{2} \psi_{0}+\frac{5}{4} r\right)^{2}+\delta^{2}}, \Pi\left(\cdot, \frac{\alpha_{1}^{2}}{\alpha_{1}^{2}-1}, k\right)
\end{aligned}
$$

is the elliptic integral of the third kind and $\operatorname{sn}(u, k)$ and $\mathrm{dn}(u, k)$ are the Jacobian elliptic functions [27]. According to (23), we may plot the graph of cuspon solution to (1) shown in Figure 4(a).

(4) Suppose that $\alpha<0,(r, \beta) \in A_{3}$. In this case, we have the phase portrait of $(8)$ shown in Figure 2(c). Notice that $H(0,0)=0$. This case is completely similar to the case of $\alpha<$ $0,(r, \beta) \in L_{3+}$.

Theorem 5. When the parameter groups $\alpha, r, \beta$ of system (8) satisfy the condition $\alpha<0$ with $r>0, \beta=-7 r^{2}$ or $\alpha<0$, $(r, \beta) \in A_{3}$, corresponding to the stable and unstable manifolds in the right phase plane of the equilibrium point $0(0,0)$ in Figure 2(m) or Figure 2(c) defined by $H(\psi, y)=0$; (1) has a cuspon solution given by (23).

Remark 6. To the best of our knowledge, solutions (23) obtained for (1) have not been reported in the literature.

3.3. Compacton. (5) Suppose that $\alpha<0,(r, \beta) \in L_{3-}$. In this case, we have the phase portrait of (8) shown in Figure 2(n). Notice that $H\left(\psi_{-}, 0\right)=H(r, 0)=-(11 / 30) r^{5}$. We see from 


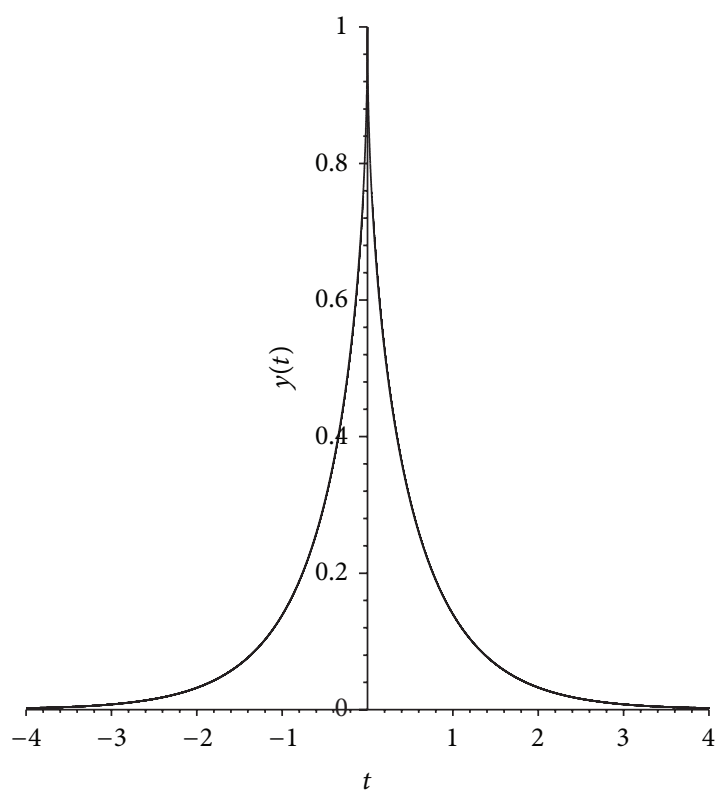

(a) $(r, \beta) \in L_{3+}$

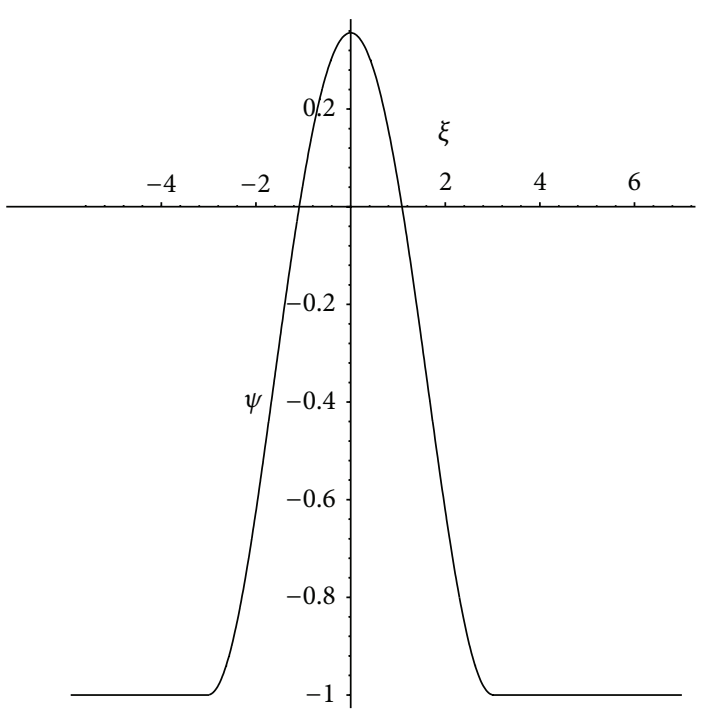

(b) $(r, \beta) \in A_{3}$

FIgURE 4: The cuspon and compacton solutions of (8) for $\alpha<0$.

(10) that arch curve connecting $A_{+}$in the right side of the straight line $\psi=r$ has the algebraic equation

$$
y^{2}=-\frac{\alpha}{5}(\psi-r)\left(\psi-\psi_{1}\right)\left(\psi-\psi_{2}\right)
$$

where $\psi_{1,2}=((-11 \pm 5 \sqrt{11 / 3}) r) / 4$. Thus, by using the first equation of (8) and (25), we obtain the parametric representation of the arch curve as follows:

$$
\psi(\xi)=\left\{\begin{array}{c}
\left(\psi_{1}\left(\psi_{2}-r\right)-\psi_{2}\left(\psi_{1}-r\right) \mathrm{sn}^{2}\right. \\
\times\left(\frac{1}{2} \sqrt{\frac{\alpha}{5}\left(r-\psi_{2}\right)} \xi, \sqrt{\left.\frac{r-\psi_{1}}{r-\psi_{2}}\right)}\right) \\
\times\left(\psi_{2}-r-\left(\psi_{1}-r\right) \mathrm{sn}^{2}\right. \\
\left.\quad \times\left(\frac{1}{2} \sqrt{\frac{\alpha}{5}\left(r-\psi_{2}\right)} \xi, \sqrt{\frac{r-\psi_{1}}{r-\psi_{2}}}\right)\right)^{-1}, \\
\quad \text { if }|\xi| \leq 2 \sqrt{\frac{-5}{2\left(\psi_{2}-r\right)}} K\left(\sqrt{\frac{r-\psi_{1}}{r-\psi_{2}}}\right), \\
\text { if }|\xi|>2 \sqrt{\frac{-5}{2\left(\psi_{2}-r\right)}} K\left(\sqrt{\frac{r-\psi_{1}}{r-\psi_{2}}}\right) .
\end{array}\right.
$$

Therefore, we have the following.

Theorem 7. (1) When the parameter groups $\alpha, r, \beta$ of system (8) satisfy the condition $\alpha<0$ with $r<0, \beta=-7 r^{2}$, there exists a homoclinic loop of system (12) given by branch of the curve $H(\psi, y)=-(11 / 30) r^{5}$.
(2) As the limit curves of a family of periodic orbits of system (8), the periodic curve in Figure 2(n) gives rise to a compacton soliton solution to (1), which has the exact parametric representation given by (26).

The profile of compacton soliton solution is shown in Figure 4(b).

Remark 8. To the best of our knowledge, solutions (26) obtained for (1) have not been reported in the literature.

3.4. Loop Solution. (6) Suppose that $\alpha<0,(r, \beta) \in L_{3-}$. We notice that the curves defined by $H(\psi, y)=h_{+}$correspond to different orbits of (8) consisting of two stable manifolds, two unstable manifolds of the saddle point $A_{+}\left(\psi_{+}, 0\right)$, and the open curve passing through the point $((7 / 2) r, 0)$, respectively (see Figure 2(n)).

We next discuss the parametric representation of $\psi(\xi)$ for these curves. We see from (10) that the arch curves in the right side of the straight line $\psi=r$ have the algebraic equation

$$
\begin{aligned}
y^{2}= & -\frac{\alpha}{5(\psi-r)^{2}} \\
& \times\left(\phi^{5}+\frac{5}{2} r \psi^{4}-\frac{35}{3} r^{2} \psi^{3}+10 r^{3} \psi^{2}-\frac{1568}{3} r^{5}\right) \\
= & -\frac{\alpha(\psi+4 r)^{2}}{5(\psi-r)^{2}}\left(\psi-\frac{7}{2} r\right)\left(\psi^{2}-2 r \psi+\frac{28}{3} r^{2}\right) .
\end{aligned}
$$

We first consider the unstable (or stable) manifold of the saddle point $A_{+}\left(\psi_{+}, 0\right)$. On the basis of Lemma 2, we can take 
initial value $\psi(0)=-(103 r / 50), y(0)=-(97 r / 573750)$ $\sqrt{276735795 \alpha r}($ or $y(0)=(97 r / 573750) \sqrt{276735795 \alpha r})$; we have from (27) that

$$
\begin{aligned}
\xi & = \pm \sqrt{-\frac{5}{\alpha}} \int_{\psi}^{\psi(0)} \Psi(\psi) d \psi \\
& = \pm \sqrt{-\frac{5}{\alpha}}\left[\int_{7 r / 2}^{\psi(0)} \Psi(\psi) d \psi-\int_{7 r / 2}^{\psi} \Psi(\psi) d \psi\right]
\end{aligned}
$$

where $\Psi(\psi)=(\psi-r) /((\psi+4 r)$ $\left.\sqrt{(\psi-(7 / 2) r)\left(\psi^{2}-2 r \psi+(28 / 3) r^{2}\right)}\right)$. By introducing a new variable $\chi$, from (28), one obtains the parametric representation of the unstable manifold:

$$
\begin{aligned}
\psi(\chi) & =\frac{(r / 2)(1-5 \sqrt{7}+(1+5 \sqrt{7}) \mathrm{cn}(\chi, k))}{1+\mathrm{cn}(\chi, k)}, \\
\xi(\chi)= & \pm \frac{5+4 \sqrt{7}}{8+3 \sqrt{7}} \sqrt{\frac{2}{5 \sqrt{7} \alpha r}} \\
& \times\left(-\chi-\Psi_{2}(\chi)+\chi_{0}+\Psi_{2}\left(\chi_{0}\right)\right),
\end{aligned}
$$

where

$$
\begin{aligned}
& \Psi_{2}(\chi)= \frac{16-6 \sqrt{7}}{3(5+4 \sqrt{7})} \\
& \times\left(\Pi\left(\arccos (\operatorname{cn}(\chi, k)), \frac{\alpha_{1}^{2}}{\alpha_{1}^{2}-1}, k\right)-\alpha_{1} f_{1}\right), \\
& \chi_{0}= \mathrm{cn}^{-1}\left(\frac{125 \sqrt{7}-278}{125 \sqrt{7}+278}, k\right), \quad \alpha_{1}=8+3 \sqrt{7} \\
& f_{1}= \frac{1}{2} \sqrt{\frac{\alpha_{1}^{2}-1}{k^{2}+k^{\prime 2} \alpha_{1}^{2}}} \\
& \times {\left[\frac{\sqrt{k^{2}+k^{\prime 2} \alpha_{1}^{2}} \mathrm{dn}(\chi, k)+\sqrt{\alpha_{1}^{2}-1}}{\sqrt{k^{2}+k^{\prime 2} \alpha_{1}^{2}} \mathrm{dn}(\chi, k)-\sqrt{\alpha_{1}^{2}-1}} \operatorname{sn}(\chi, k)\right] } \\
& k^{2}=\sqrt{\frac{1}{2}}\left(1+\frac{1}{7} \sqrt{7}\right)
\end{aligned}
$$

In addition, taking the initial condition $\psi(0)=7 r / 2$, $y(0)=0$, for the left open curve defined by a branch of $H(\psi, y)=h_{+}$, we have the parametric representation:

$$
\begin{gathered}
\psi(\chi)=\frac{(r / 2)(1-5 \sqrt{7}+(1+5 \sqrt{7}) \mathrm{cn}(\chi, k))}{1+\operatorname{cn}(\chi, k)}, \\
\xi(\chi)= \pm \frac{5+4 \sqrt{7}}{8+3 \sqrt{7}} \sqrt{\frac{2}{5 \sqrt{7} \alpha r}}\left(\chi+\Psi_{2}(\chi)\right) .
\end{gathered}
$$

Employing the above formulas to draw the graphs of $\psi(\xi)$, we obtain corresponding wave profiles shown in Figure 5.
Remark 9. The loop solution, that is, the so-called loop soliton solution, is not one real soliton solution (see $[28,29])$.

(7) Suppose that $\alpha<0,(r, \beta) \in A_{4}$. We notice that the curves defined by $H(\psi, y)=0$ correspond to different orbits of (8) consisting of two stable manifolds, two unstable manifolds of the saddle point $O(0,0)$, and the open curve passing through the point $\left(\psi_{2}, 0\right)$, respectively (see Figure 2(d)).

We next discuss the parametric representation of $\psi(\xi)$ for these curves. We see from (10) that the arch curves in the left side of the straight line $\psi=r$ have the algebraic equation

$$
\begin{aligned}
y^{2} & =-\frac{\alpha \psi^{2}}{5(\psi-r)^{2}}\left(\phi^{3}+\frac{5}{2} r \psi^{2}+\frac{5}{3} \beta \psi-\frac{5}{2}\left(3 r^{2}+\beta\right)\right) \\
& =-\frac{\alpha \psi^{2}}{5(\psi-r)^{2}}\left(\psi-\psi_{1}\right)\left(\psi-\psi_{2}\right)\left(\psi-\psi_{3}\right),
\end{aligned}
$$

where $\psi_{1}<0<\psi_{2}<\psi_{3}$.

We first consider the unstable (or stable) manifold of the saddle point $O(0,0)$. On the basis of Lemma 2, we can take initial value

$$
\begin{aligned}
& \psi(0)=-\frac{11 r}{25} \\
& y(0)=\sqrt{-\frac{121 \alpha r}{980}\left(\frac{17787 r^{2}}{31250}+\frac{11 \beta}{15}-\frac{5\left(3 r^{2}+\beta\right)}{2}\right)} \\
& \left(\begin{array}{l}
\text { or } \\
y(0) \\
\left.=-\sqrt{-\frac{121 \alpha r}{980}\left(\frac{17787 r^{2}}{31250}+\frac{11 \beta}{15}-\frac{5\left(3 r^{2}+\beta\right)}{2}\right)}\right)
\end{array}\right.
\end{aligned}
$$

We have from (32) that

$$
\xi= \pm \sqrt{-\frac{5}{\alpha}} \int_{\psi(0)}^{\psi} \frac{(\psi-r) d \psi}{\psi \sqrt{\left(\psi-\psi_{1}\right)\left(\psi-\psi_{2}\right)\left(\psi-\psi_{3}\right)}} .
$$

By introducing a new variable $\chi$, from (34), one obtains the parametric representation of the unstable manifold:

$$
\begin{aligned}
\psi(\chi) & =\frac{\psi_{2}\left(\psi_{3}-\psi_{1}\right)-\psi_{3}\left(\psi_{2}-\psi_{1}\right) \operatorname{sn}^{2}(\chi, k)}{\left(\psi_{3}-\psi_{1}\right)-\left(\psi_{2}-\psi_{1}\right) \operatorname{sn}^{2}(\chi, k)} \\
\xi(\chi)= & \pm 2 \sqrt{\frac{5}{-\alpha\left(\psi_{3}-\psi_{1}\right)}} \\
& \times\left(\left(1-\frac{r}{4}\right) \chi+\Psi_{3}(\chi)-\left(1-\frac{r}{4}\right) \chi_{0}-\Psi_{3}\left(\chi_{0}\right)\right)
\end{aligned}
$$




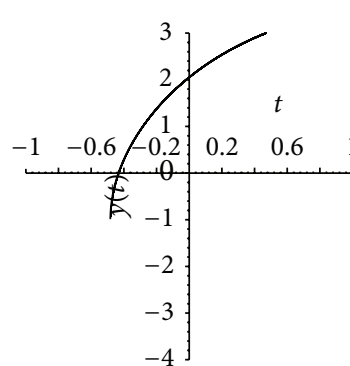

(a)

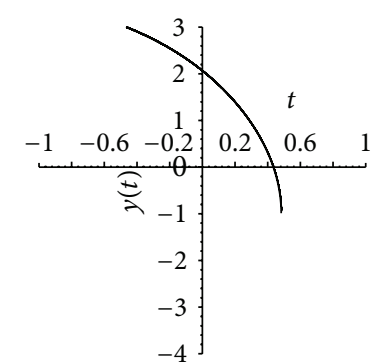

(b)

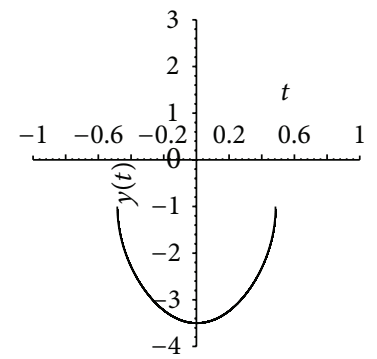

(c)

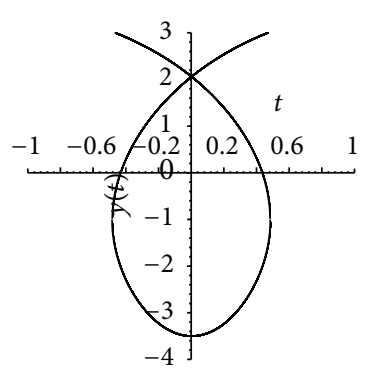

(d)

Figure 5: The profiles of waves when $\alpha<0,(r, \beta) \in L_{3-}$. (a) shows the kink wave corresponding to the unstable manifold. (b) shows the kink wave corresponding to the stable manifold. (c) shows the breaking wave corresponding to the open curve passing through the point $A_{+}\left(\psi_{+}, 0\right)$. (d) shows the wave profile of three curves-loop solution.

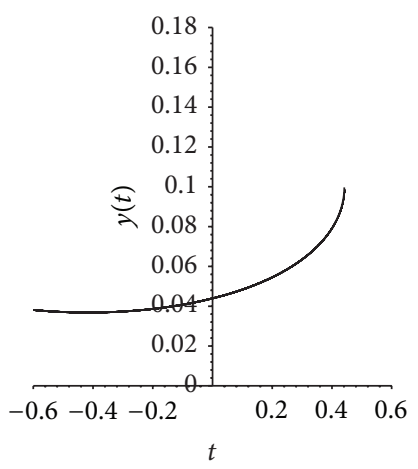

(a)

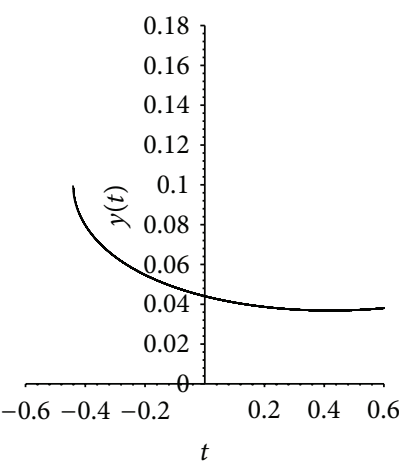

(b)

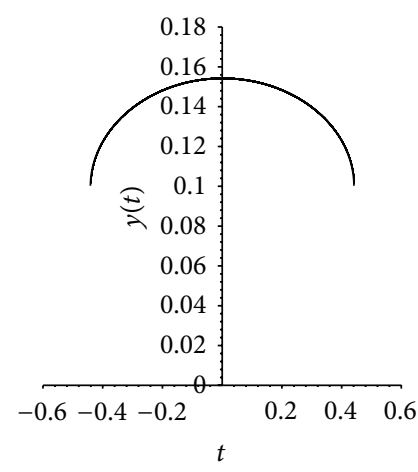

(c)

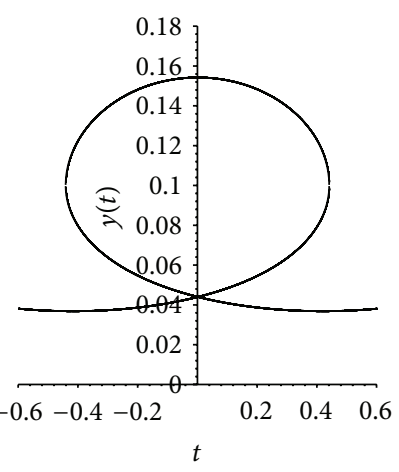

(d)

FIGURE 6: The profiles of waves when $\alpha<0,(r, \beta) \in A_{4}$. (a) shows the kink wave corresponding to the unstable manifold. (b) shows the kink wave corresponding to the stable manifold. (c) shows the breaking wave corresponding to the open curve passing through the point $O(0,0)$. (d) shows the wave profile of three curves-loop solution.

where

$$
\begin{gathered}
\Psi_{3}(\chi)=-\frac{r\left(\psi_{2}-\psi_{1}\right)^{2}}{\psi_{2}\left(\psi_{3}-\psi_{1}\right)}\left(\Pi\left(\arcsin (\operatorname{sn}(\chi, k)), \alpha_{1}^{2}, k\right)\right), \\
\chi_{0}=\operatorname{sn}^{-1}\left(\sqrt{\frac{\left(\psi_{3}-\psi_{1}\right)\left(\psi_{2}-\psi(0)\right)}{\left(\psi_{2}-\psi_{1}\right)\left(\psi_{3}-\psi(0)\right)}}, k\right), \\
\alpha_{1}=\frac{\psi_{3}\left(\psi_{2}-\psi_{1}\right)}{\psi_{2}\left(\psi_{3}-\psi_{1}\right)}, \quad k^{2}=\frac{\psi_{2}-\psi_{1}}{\psi_{3}-\psi_{1}} .
\end{gathered}
$$

In addition, taking the initial condition $\psi(0)=\psi_{2}, y(0)=$ 0 , for the right open curve defined by a branch of $H(\psi, y)=0$, we have the parametric representation:

$$
\begin{gathered}
\psi(\chi)=\frac{\psi_{2}\left(\psi_{3}-\psi_{1}\right)-\psi_{3}\left(\psi_{2}-\psi_{1}\right) \mathrm{sn}^{2}(\chi, k)}{\left(\psi_{3}-\psi_{1}\right)-\left(\psi_{2}-\psi_{1}\right) \mathrm{sn}^{2}(\chi, k)}, \\
\xi(\chi)= \pm 2 \sqrt{\frac{5}{-\alpha\left(\psi_{3}-\psi_{1}\right)}}\left(\left(1-\frac{r}{4}\right) \chi+\Psi_{3}(\chi)\right) .
\end{gathered}
$$

Employing the above formulas to draw the graphs of $\psi(\xi)$, we obtain corresponding wave profiles shown in Figure 6.
Finally, we note that in Figures 2(e), 2(f), 2(g), 2(h), and $2(1)$, there is at least one loop solution to (1). Due to space limitations, we omit them.

Remark 10. To the best of our knowledge, solutions (31) and (37) obtained for (1) have not been reported in the literature.

\section{Discussion}

In this paper, we used the qualitative analysis methods of a dynamical system to investigate the peakon, cuspon, compacton, and loop solutions of $\operatorname{3DKP}(3,2)$ equation. Our procedure shows that the $\operatorname{3DKP}(3,2)$ equation either has peakon, cuspon, compacton, or loop solutions. The phase portrait bifurcation of the traveling wave system corresponding to the equation is given. Particularly, cuspon and loop belong to the compound-type solutions; that is, they consist of two or three branches of nonsmooth solutions due to the existence of the singular straight line $\psi=r$ in the corresponding phase portraits. The approach we used is simple and can be extended to study the soliton solutions of some other equations. 


\section{Conflict of Interests}

The authors declare that there is no conflict of interests regarding the publication of this paper.

\section{Acknowledgments}

The authors wish to thank the anonymous reviewers for their helpful comments and suggestions. This work is supported by the National Natural Science Foundation of China (nos. 11361017 and 11161013) and Natural Science Foundation of Guangxi (nos. 2012GXNSFAA053003 and 2013GXNSFAA019010).

\section{References}

[1] M. J. Ablowitz and P. A. Clarkson, Solitons, Nonlinear Evolution Equations and Inverse Scattering, vol. 149 of London Mathematical Society Lecture Note Series, Cambridge University Press, Cambridge, UK, 1991.

[2] V. B. Matveev and M. A. Salle, Darboux Transformation and Solitons, Springer, Berlin, Germany, 1991.

[3] C. H. Gu, H. S. Hu, and Z. X. Zhou, Darboux Transformations in Soliton Theory and Its Geometric Applications, Shanghai Science and Technology, Shanghai, China, 1999.

[4] R. Hirota and J. Satsuma, "Soliton solutions of a coupled Korteweg-de Vries equation," Physics Letters A, vol. 85, no. 8-9, pp. 407-408, 1981.

[5] P. J. Olver, Applications of Lie Groups to Differential Equations, Springer, New York, NY, USA, 1993.

[6] G. W. Bluman and S. Kumei, Symmetries and Differential Equations, Springer, Berlin, Germany, 1989.

[7] J. Li and Z. Liu, "Traveling wave solutions for a class of nonlinear dispersive equations," Chinese Annals of Mathematics B, vol. 23, no. 3, pp. 397-418, 2002.

[8] M. Z. Wei, S. Q. Tang, H. L. Fu, and G. X. Chen, "Single peak solitary wave solutions for the generalized KP-MEW $(2,2)$ equation under boundary condition," Applied Mathematics and Computation, vol. 219, no. 17, pp. 8979-8990, 2013.

[9] A. Wazwaz, "A reliable treatment of the physical structure for the nonlinear equation $K(m, n)$," Applied Mathematics and Computation, vol. 163, no. 3, pp. 1081-1095, 2005.

[10] A.-M. Wazwaz, "Analytic study on nonlinear variants of the RLW and the PHI-four equations," Communications in Nonlinear Science and Numerical Simulation, vol. 12, no. 3, pp. 314-327, 2007.

[11] Z. Yan and H. Zhang, "New explicit and exact travelling wave solutions for a system of variant Boussinesq equations in mathematical physics," Physics Letters A, vol. 252, no. 6, pp. 291296, 1999.

[12] Z. Yan, "New explicit travelling wave solutions for two new integrable coupled nonlinear evolution equations," Physics Letters A, vol. 292, no. 1-2, pp. 100-106, 2001.

[13] E. Fan, "Uniformly constructing a series of explicit exact solutions to nonlinear equations in mathematical physics," Chaos, Solitons and Fractals, vol. 16, no. 5, pp. 819-839, 2003.

[14] S. Zhang, "New exact solutions of the KdV-Burgers-Kuramoto equation," Physics Letters A, vol. 358, no. 5-6, pp. 414-420, 2006.

[15] M.-L. Wang, "Exact solutions for a compound KdV-Burgers equation," Physics Letters A, vol. 213, no. 5-6, pp. 279-287, 1996.
[16] F. Xie and Z.-Y. Yan, "Compactons and noncompactons to three-dimensional Kadomtsev-Petviashvili equation with nonlinear dispersion," Chaos, Solitons \& Fractals, vol. 36, no. 2, pp. 278-282, 2008.

[17] E. A. Kuznetsov and S. K. Turitsyn, "Two-and three-dimensional solitons in weakly dispersive media," Zhurnal Eksperimental'noi i Teoreticheskoi Fiziki, vol. 82, pp. 1457-1463, 1982.

[18] E. A. Kuznietsov and S. L. Musher, "Effect of collapse of sound waves on the structure of collisionless shock waves in a magnetized plasma," Journal of Experimental and Theoretical Physics, vol. 64, no. 5, pp. 947-955, 1986.

[19] P. Rosenau and J. M. Hyman, "Compactons: solitons with finite wavelength," Physical Review Letters, vol. 70, no. 5, pp. 564-567, 1993.

[20] M. Inc, "Compact and noncompact structures of a threedimensional 3DKP $(m, n)$ equation with nonlinear dispersion," Applied Mathematics Letters, vol. 26, no. 4, pp. 437-444, 2013.

[21] S. N. Chow and J. K. Hale, Method of Bifurcation Theory, Springer, New York, NY, USA, 1981.

[22] J. Li and Z. Liu, "Smooth and non-smooth traveling waves in a nonlinearly dispersive equation," Applied Mathematical Modelling, vol. 25, no. 1, pp. 41-56, 2000.

[23] Y. Xie, H. Fu, and S. Tang, "Peaked and smooth solitons for $K *(4,1)$ equation," Journal of Applied Mathematics, vol. 2013, Article ID 518415, 10 pages, 2013.

[24] H. Fu, Y. Tang, S. Tang, H. Yan, and Q. Liu, "Peaked and smooth solitons for $K^{*}(3,1)$ equation, Indian," Journal of Physics, vol. 88, pp. 83-91, 2014.

[25] J. Li and Z. Qiao, "Peakon, pseudo-peakon, and cuspon solutions for two generalized Camassa-Holm equations," Journal of Mathematical Physics, vol. 54, no. 12, Article ID 123501, 14 pages, 2013.

[26] R. Camassa and D. D. Holm, "An integrable shallow water equation with peaked solitons," Physical Review Letters, vol. 71, no. 11, pp. 1661-1664, 1993.

[27] P. F. Byrd and M. D. Friedman, Handbook of Elliptic Integrals for Engineers and Scientists, Springer, Berlin, Germany, 1971.

[28] J. B. Li, Singular Nonlinear Travelling Wave Equations: Bifurcations and Exact Solutions, Science Press, Beijing, China, 2013.

[29] J. Li, "Dynamical understanding of loop soliton solution for several nonlinear wave equations," Science in China. Series A. Mathematics, vol. 50, no. 6, pp. 773-785, 2007. 


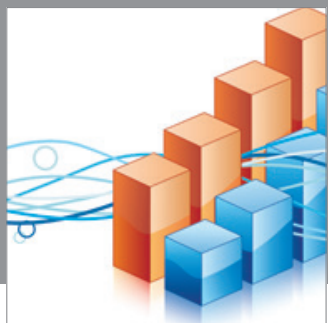

Advances in

Operations Research

mansans

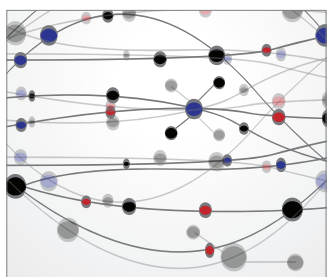

The Scientific World Journal
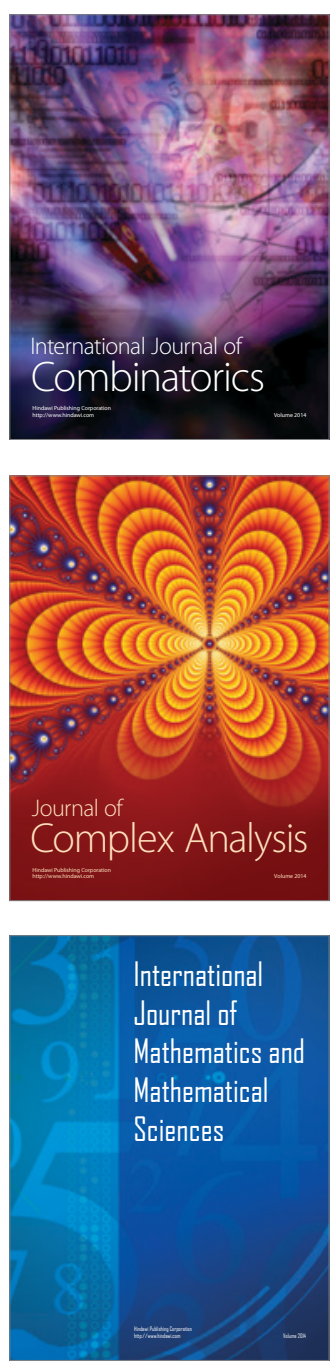
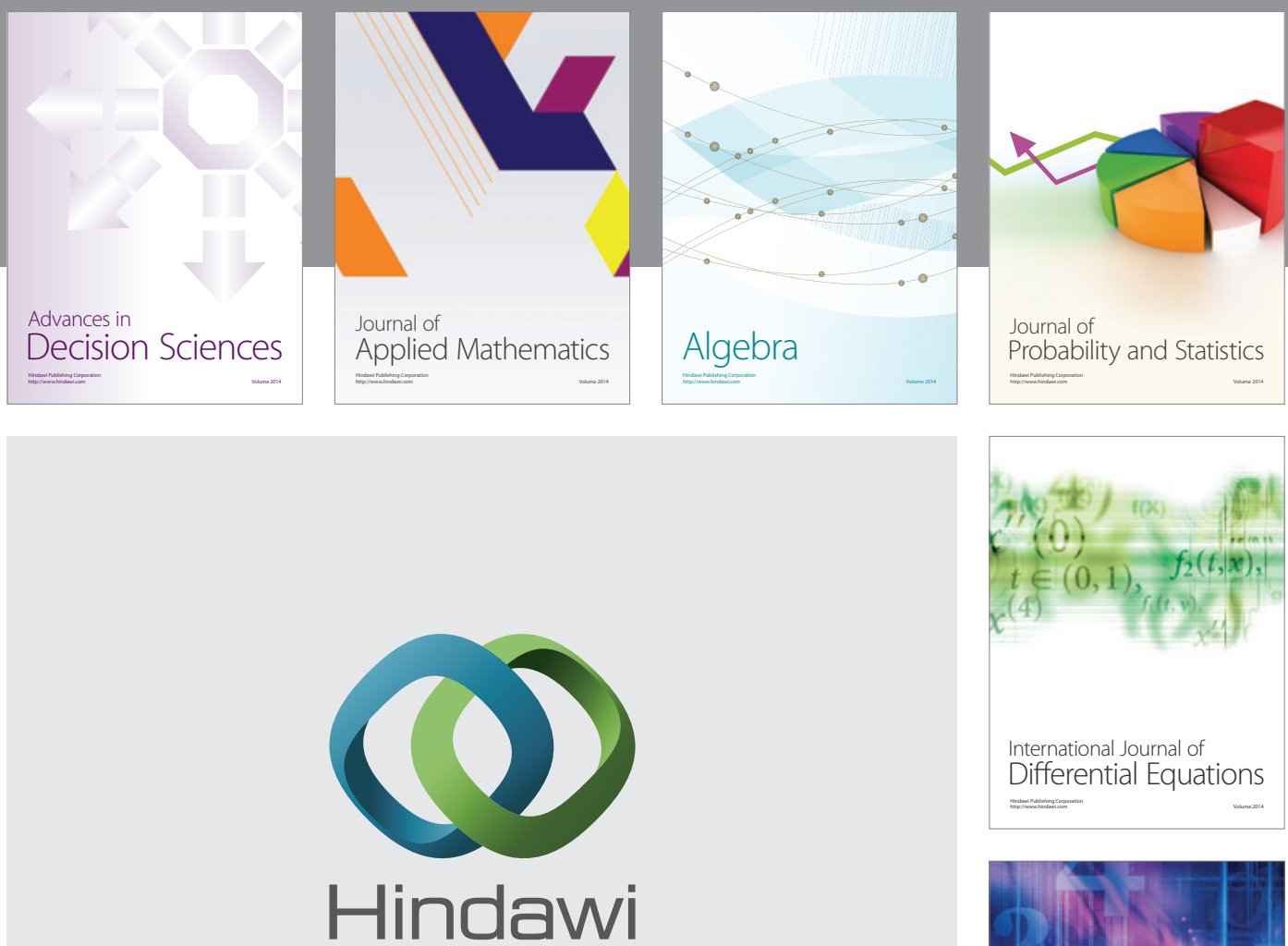

Submit your manuscripts at http://www.hindawi.com
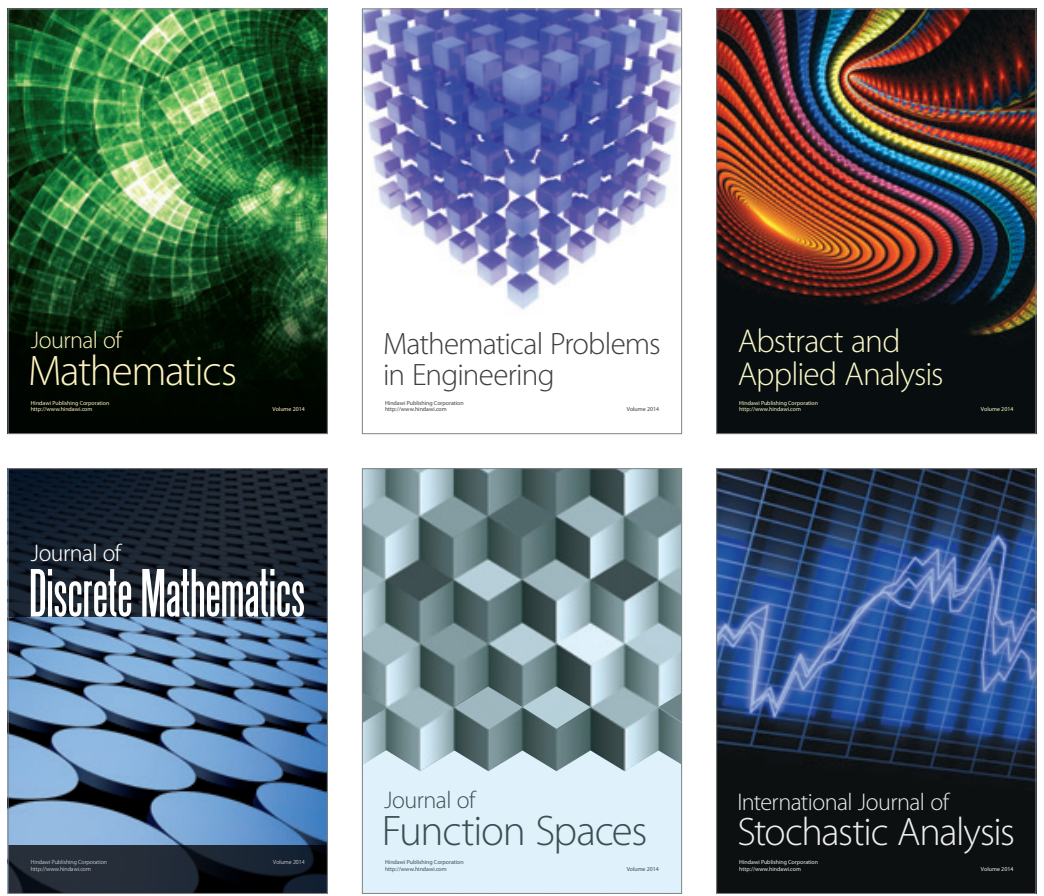

Journal of

Function Spaces

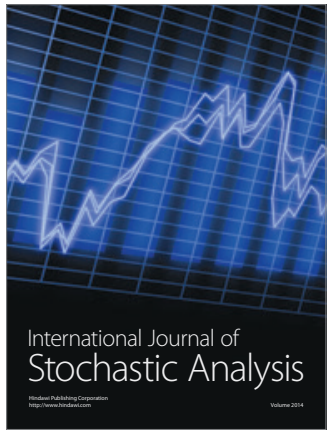

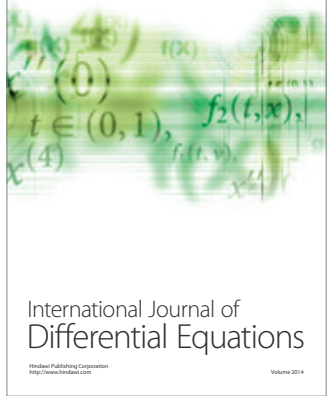
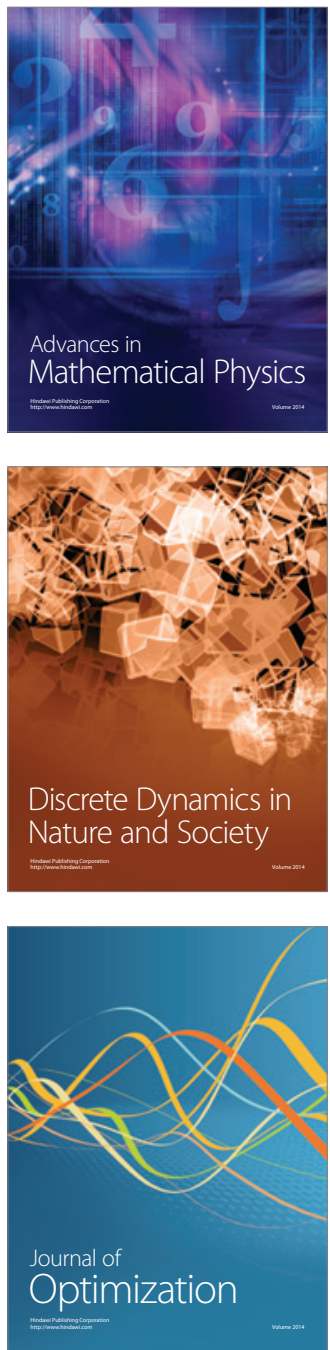